\title{
Sideband Separating Mixer for 600-720 GHz
}

\author{
Andrey Khudchenko*a, Ronald Hesper ${ }^{\mathrm{b}}$, Andrey Baryshev ${ }^{\mathrm{a}, \mathrm{b}}$, Gerrit Gerlofma ${ }^{\mathrm{b}}$, Patricio Mena ${ }^{\mathrm{c}}$, \\ Tony Zijlstra ${ }^{\mathrm{d}}$, Teun Klapwijk ${ }^{\mathrm{d}}$, Marco Spaans ${ }^{\mathrm{b}}, \mathrm{Jacob}_{\mathrm{W}}$ W. Kooi ${ }^{\mathrm{e}}$ \\ ${ }^{a}$ Netherlands Institute for Space Research, Landleven 12, 9747 AD Groningen, The Netherlands \\ ${ }^{b}$ Kapteyn Astronomical Institute, Landleven 12, 9747 AD Groningen, The Netherlands \\ ${ }^{c}$ Electrical Engineering Department, Universidad de Chile, Av. Tupper 2007, Santiago, Chile \\ ${ }^{\mathrm{d} K a v l i}$ Institute of Nanoscience, Delft University of Technology, Lorentzweg 1, 2628 CJ Delft \\ The Netherlands \\ ${ }^{\mathrm{e}}$ California Institute of Technology, MS 320-47 Pasadena, CA 91125, USA
}

\begin{abstract}
The ALMA Band 9 receiver cartridge (600-720 GHz) based on Dual Sideband (DSB) superconductor-insulatorsuperconductor (SIS) mixer is currently in full production. In the case of spectral line observations, the integration time to reach a certain signal-to-noise level can be reduced by about a factor of two by rejecting an unused sideband. The goal is to upgrade the current ALMA band 9 cartridge to a full dual-polarization sideband separating (2SB) capability, with minimal-cost upgrade path. A new compact and modular sideband separating mixer was designed, and a prototype manufactured. The individual SIS mixer devices in the 2SB block are implemented as conventional Band 9 DSB mixers, so that existing devices can be reused and tested individually. Any ALMA DSB developments contribute to the 2SB upgrade. The first experimental results demonstrate noise temperature from $300 \mathrm{~K}$ to $500 \mathrm{~K}$ over $80 \%$ of the band, which will be improved to fit the ALMA requirements. Nevertheless, the frequency response for 2SB is the same as for DSB, showing that the RF design is still valid, even with different SIS mixer devices. The quality of the RF and IF design is confirmed by a sideband rejection ratio of about $15 \mathrm{~dB}$, which is within the ALMA spec (>10dB ).
\end{abstract}

Keywords: Sideband separating mixers, Submillimeter mixers, ALMA band 9, superconductor-insulator-superconductor junction

\section{INTRODUCTION}

Especially for high frequency bands like band 9, this is a significant handicap because of the relatively high absorption (and therefore emission) of the atmosphere, even under the best of observing conditions. Removing the atmospheric noise from the image band can reduce the required observation time by a factor of about two, depending on the relative intensities of the spectral lines and the background. The new design is targeted to facilitate a minimal-impact upgrade of the existing band 9 receiver cartridge with sideband separating (2SB) mixers, but other applications can be considered as well.

Here we report the first results on the RF and IF performance of a new 2SB mixer. This mixer has a new modular design with a possibility to test SIS junctions individually before mounting in the 2SB mixer block, and to exchange them independently.

*A.Khudchenko@sron.nl; phone +31 50363 4074; fax +31 50363 4033; sron.nl

Infrared, Millimeter Wave, and Terahertz Technologies, edited by Cunlin Zhang,

Xi-Cheng Zhang, Peter H. Siegel, Li He, Sheng-Cai Shi, Proc. of SPIE Vol. 7854, 78542R

(C) 2010 SPIE · CCC code: $0277-786 X / 10 / \$ 18 \cdot$ doi: $10.1117 / 12.869594$

Proc. of SPIE Vol. $785478542 R-1$ 


\section{CONCEPT AND MECHANICAL DESIGN}

\subsection{Concept}

Several years ago, an effort was initiated to investigate the possibility to provide 2SB mixers for ALMA band 9 [1], [2]. This mixer was a monolithic splitblock design containing the RF hybrid, Local Oscillator (LO) couplers, LO horn, matched loads, SIS junctions, magnet coils and magnet conductors for Josephson suppression and DC bias tees. The corrugated RF input horn was bolted as a separate component to the mixer block.

One of the main lessons learned from the implementations of the original 2SB mixer block is the importance of a modular design. In the first place, because of the high complexity of the mixer block, involving machining on vastly different scales, it turned out to be very difficult to obtain blocks of satisfactory quality. Many times, in a late stage of production, small mistakes or accidents happened, ruining all the machining effort that had been put in already. In a more modular design, production is still critical, but the consequences of errors will be much more contained.

Another serious handicap that turned up in the monolithic design was the inability to replace SIS mixer devices individually. To obtain a high sideband separation, not only the hybrids have to be of high quality, also the junctions (especially their gain) must be balanced as much as possible. In the new design it is possible to exchange the SIS mixer devices independently.

\subsection{Modular hybrid - mixer block}

Since the current production of single-ended mixers for the ALMA band 9 cartridges involves testing and characterization of many SIS devices, it was decided to design the new 2SB mixer block around the standard band 9 junction holders ("backpieces"). These are compact, easy to mount on a standard horn for testing and matching, reusable, and, because of the high production volume, relatively cheap. The mounting and unmounting of mixer substrates is routinely done in-house. And, finally, since the junctions are in the same RF environment as the single-ended band 9 mixers, no new junction design is necessary; in fact, the 2SB mixer development effort benefits immediately from improvements and developments in the current production devices.

Two band 9 backpieces mate to the waveguides coming from the LO couplers. The alignment of the waveguides to the backshort cavity in the backpieces is done by a centering ring, a proven concept used in the single-ended band 9 mixers. It allows for easy exchange of the SIS mixer devices without need of any special tools or even a microscope. The backpieces are held in place by spring-loaded retainer caps, also adapted from the DSB mixers. The magnet coils for the suppression of the Josephson current are integrated into the backpiece covers, together with the magnetic field conductors that guide the field to the junction. Figure 1 left part shows the cross section of the hybrid and write part how the assembly is done. A few clips mounted on the top and bottom of the blocks provide pressure to the field conductors and the cable-mounted GPO IF connectors.

The RF hybrid and LO injecting structure is based on the design made for previous 2SB mixer block concept [1], [2], therefore special transitions to fit the waveguides height to the full height of the standard ALMA horns is made.
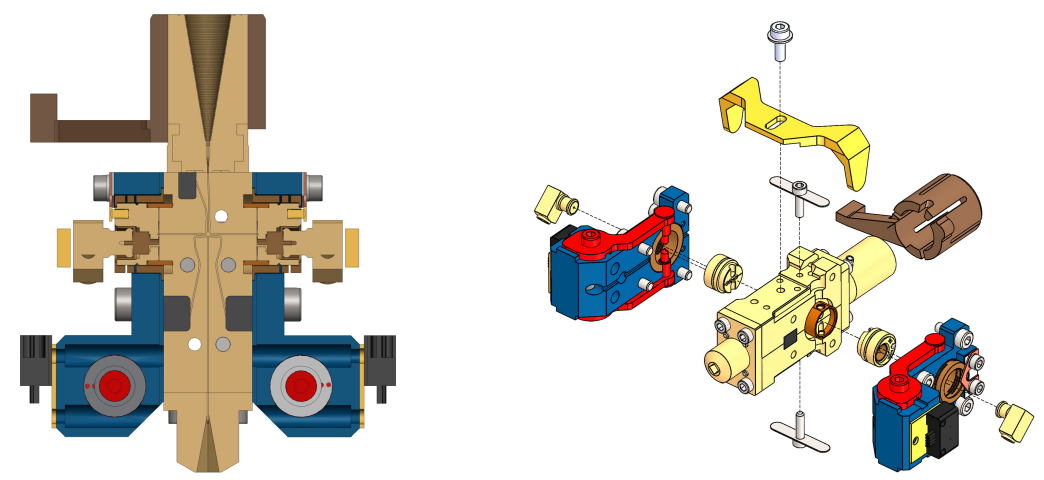

Figure 1. Left picture: cross-section of the new 2SB mixer block. The RF input horn is at the top, the LO horn at the bottom. Left and right of the hybrid are two standard ALMA band 9 mixer backpieces, below them the magnet coils. Right picture: exploded view of the new 2SB mixer, showing the way it is disassembled to replace the mixer backpieces. 


\section{EXPERIMENTAL RESULTS}

Since the new design uses standard ALMA band 9 back pieces for the SIS junctions, the RF response of each SIS junction could be measured before the installation in the 2SB mixer block. By comparing the results with those of the complete $2 \mathrm{SB}$ mixer, the flatness of the RF response can be investigated.

The properties of the RF hybrid and LO splitter were studied in a direct way, by measuring the balance of pumping levels, both through the LO port and the RF port. The frequency response was determined by a Fourier transform spectrometer (FTS). Finally, the noise temperature and sideband rejection ratio were measured.

Some additional properties were investigated to determine the operational characteristics of the design. The magnetic cross talk between the Josephson suppression coils turned out to be about $4 \%$, which is an acceptable level. Also, the thermal cross talk between the deflux heaters is small enough to allow defluxing one mixer without the other one going out of superconductivity.

\subsection{RF Hybrid Characterization}

The direct response, as a function of frequency, of both SIS junctions contained in the 2SB mixer has been measured using a Fourier transform spectrometer, both individually and in the 2SB block. The results are presented in the figure 2 .

The FTS spectra show that ALMA band $9(600-720 \mathrm{GHz})$ is covered as intended. Comparing the "2SB mode" spectra (solid lines) with the ones taken with the individual SIS mixer devices (DSB mode, dashed lines) shows that the frequency response is not dramatically influenced, neither by the 2SB RF structure itself, nor by the full height to reduced height waveguide transitions.
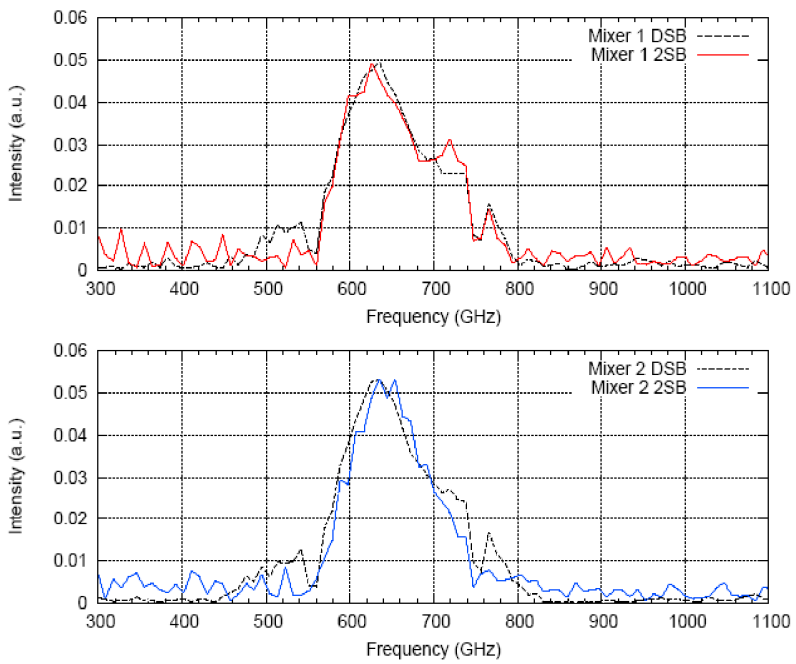

Figure 2. Direct RF response of the SIS junctions: dashed lines - individual; solid lines - response of the junctions installed in the hybrid block.

Figure 3 characterizes the balance of the RF hybrid and LO splitter. Ideally, both should be perfect $-3 \mathrm{~dB}$ splitters, the former with an additional 90 degree phase shift and the latter in-phase. Since the pumping current of an SIS junction at a fixed bias point is proportional to the RF power reaching the junction [3], the ratio of the currents is equal to the power ratio, independent of frequency or absolute LO power.

The curve measured with the signal injected through the LO port shows a periodic frequency dependent misbalance in the LO distribution structure (LO splitter, couplers and dummy loads), indicating that there may be a standing wave. The period correspond to a half wavelength of about $10 \mathrm{~mm}$ and are probably caused by reflection from the LO loads. This imbalance prevents both mixers to be pumped optimally at the same time. Since, around the optimal pumping level, the noise performance of an SIS mixer is not very strongly depending on LO power, deviations less than about $20 \%$ are 
acceptable. So, the LO coupling ratio, shown in figure 3, is not too far from being acceptable, but the real impact should be judged from noise temperature and sideband ratio measurements.

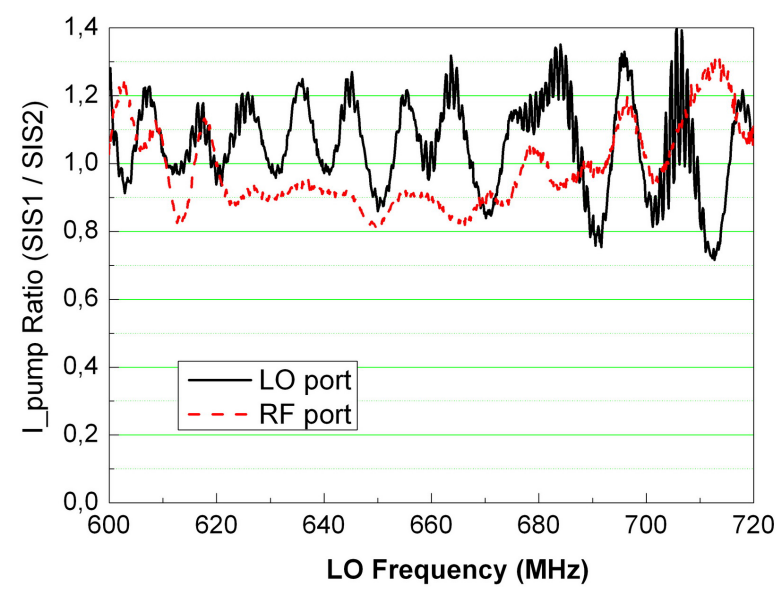

Figure 3. Ratio of pumping currents of the SIS junctions at the signal injection through the RF or LO ports.

\subsection{IF hybrid characterization}

The initial tests were performed with 4-8 GHz cryogenic amplifiers. This range will be extended soon to the $4-12 \mathrm{GHz}$ by installing standard Yebes ALMA band 9 cryogenic amplifiers. The 90 degree IF hybrid (provided by Observatorio Astronomico Nacional, Guadalajara,Spain, described in [4]) is placed between the SIS junctions and the 4-12 GHz isolators before the amplifiers. The hybrid has amplitude unbalance less than $\pm 0.3 \mathrm{~dB}$ and phase unbalance not exceeding \pm 2 degrees, over the 4-12 GHz band.

The performance of the entire IF chain was determined, in a standard way, by using the SIS junctions as noise sources, biasing them at different voltages ( 4 and $10 \mathrm{mV}$ in this case). Since the level of the noise generated by a SIS junctions is known we measure the IF output in three cases: 4 and $4 \mathrm{mV}$; 4 and $10 \mathrm{mV} ; 10$ and $4 \mathrm{mV}$ (for the first and the second SIS junctions correspondingly); and from these curves we calculate the ratio between 90 degree branch and direct one coming to LSB or USB ports. The result of calculation is presented on the figure 4 (left graph). In case the deviation of the presented curves from 1 is less than $25 \%$ (about $1 \mathrm{~dB}$ misbalance) it should not be a significant influence on the sideband rejection ratio.

By biasing both SIS junctions to $4 \mathrm{mV}$ first and to $10 \mathrm{mV}$ afterwards the noise temperatures of the IF chains were calculated. It's level is less than $5 \mathrm{~K}$ in the presented IF band (see figure 4 right graph).
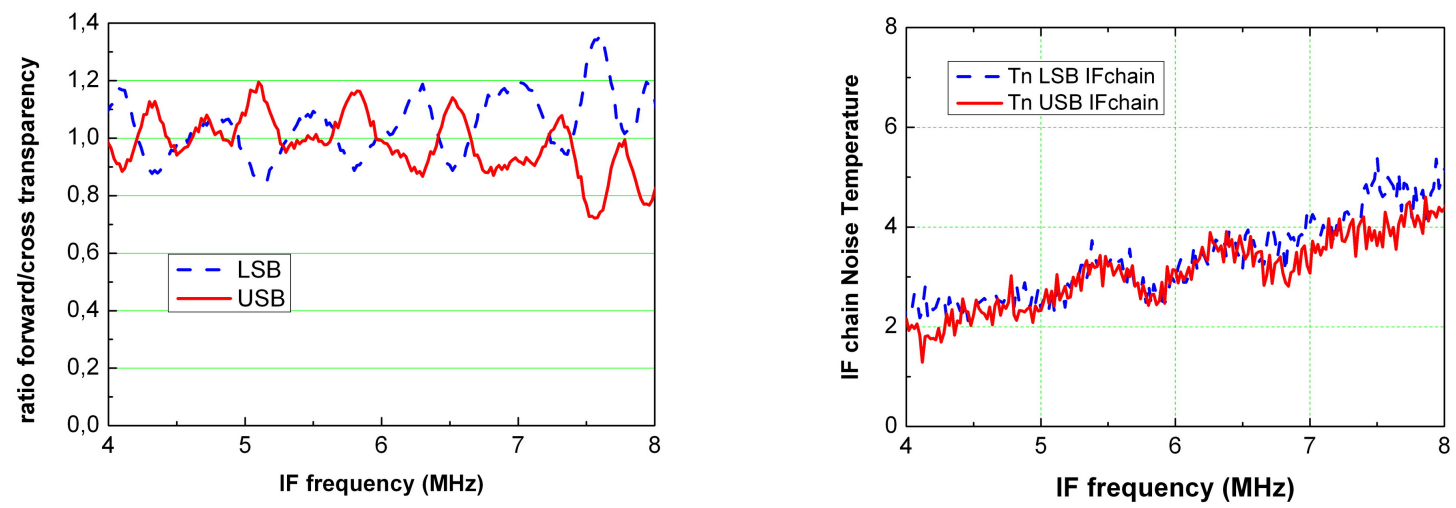

Figure 4. Test of the USB and LSB IF chain by biasing SIS junctions to 4 and $10 \mathrm{mV}$. 


\subsection{Noise temperature and sideband rejection ratio}

The noise temperature was measured using the conventional Y-factor method. The results for both lower sideband (LSB) and upper sideband (USB) IF outputs are summarized in the Figure 5. The best noise temperature obtained is about $300 \mathrm{~K}$ (SSB). There is also plotted in figure 5 the DSB noise temperature of one of the individual junctions, converted to a corresponding ideal SSB noise temperature by scaling it with a factor 2. This way, the shape of the expected curve (for the case of perfect hybrids) can be compared to the actual measurement. As visible in the figure, the measured data follows the one expected from the DSB data rather closely in shape. However, the overall level of the noise is about $50 \%$ higher than expected.

The ALMA specifications for single-sideband noise are indicated by the horizontal lines in figure 5: $80 \%$ of the band should not exceed $335 \mathrm{~K}$ while all points should below $500 \mathrm{~K}$ [5]. In these initial measurements, the noise performance is still higher than required. The investigation of the causes of this increased noise temperature is ongoing at the time of writing.

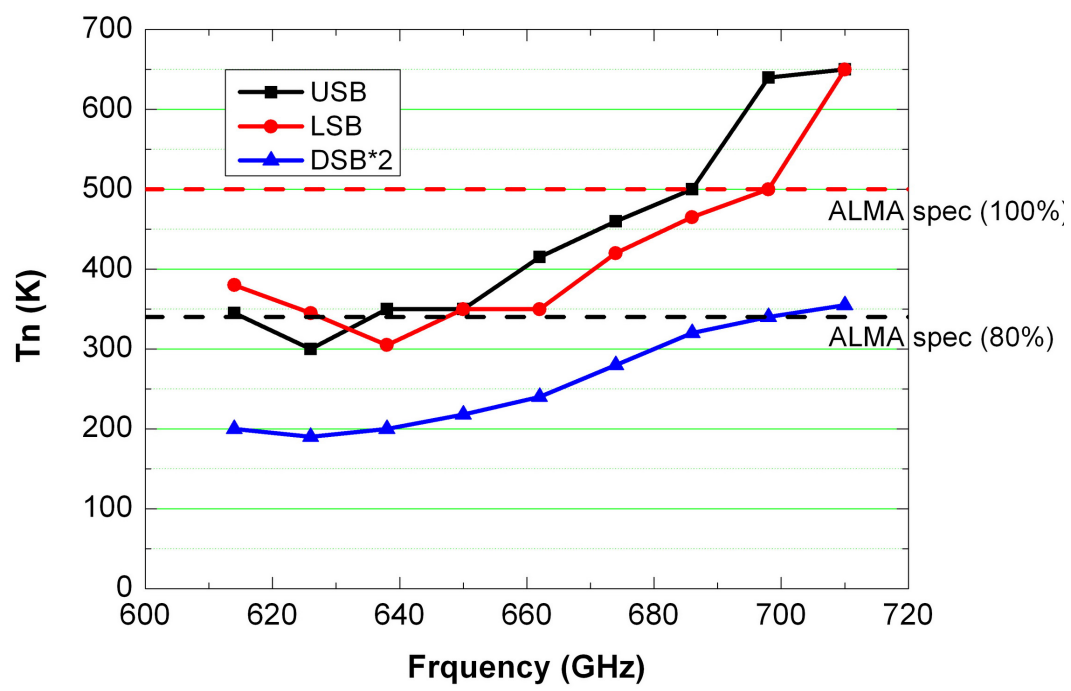

Figure 5. Single sideband noise temperatures at different LO frequencies. Colored horizontal lines represent ALMA specifications. The curve labeled DSB*2 represents the DSB noise temperature of one of the individual SIS devices, scaled to show the expected ideal SSB performance.

The sideband ratio (power in USB / power in LSB) was determined by injecting a small test signal, through a 6 um mylar beamsplitter, into the RF port of the receiver, and taking spectra of both IF output signals. Figure 6 shows a rough indication of the sideband rejection ratios. It consists of superimposed IF spectra, taken with different frequencies of the test signal. The individual IF spectra are corrected for the static baseline of the pumped IF signal without test tone, and the USB signal is divided by the LSB signal and plotted in dBs. With the test signals in the upper sideband (4-8 GHz above the LO frequency), we now get a positive ratio (upper panel) and in the lower sideband (4-8 GHz below LO) negative (lower panel). This was done for a single LO frequency $(662 \mathrm{GHz})$, about in the middle of the band. The sideband ratio is now approximately given by the line connecting the tops of the IF spectra. A more careful analysis, following [6] will be performed in the future when more data is available. Nevertheless, it seems that ALMA spec for the sideband suppression $(10 \mathrm{~dB})$ is already reached. 


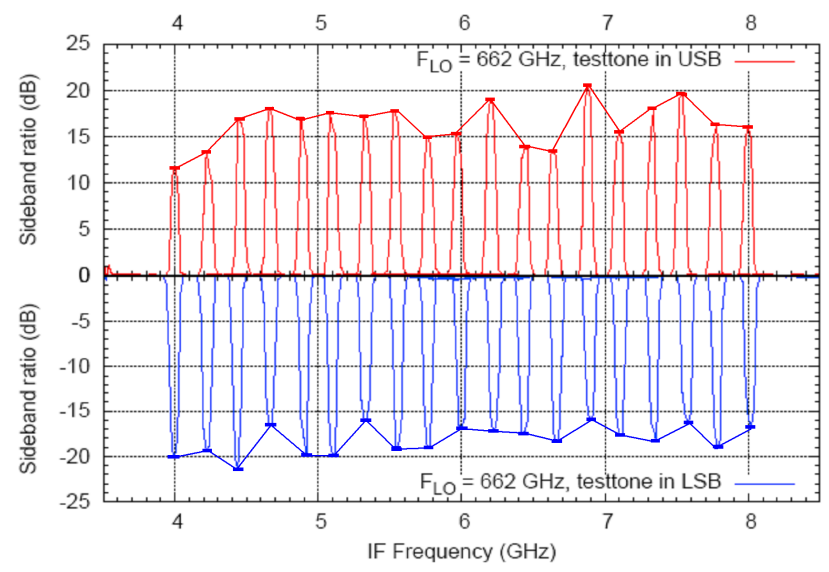

Figure 6. Sideband ratios for upper sideband (red curve) and for lower sideband (blue curve). See text for explanation.

\section{ACKNOWLEDGEMENTS}

The authors thank Joost Adema for help with organizing of the experiment. This effort/activity is supported in part: by the European Community Framework Program 7, Advanced Radio Astronomy in Europe, grant agreement no. 227290; Dutch NWO/STW VENI Grant 08119, "Advanced Heterodyne Mixers for THz Applications", Dutch research school for astronomy (NOVA) NOVAIII grant.

\section{REFERENCES}

[1] Mena, F. P., Kooi, J. W., Baryshev, A. M., Lodewijk, C. F. J., Hesper, R., Wild, W. and Klapwijk, T. M., "Construction of a Side Band Separating Heterodyne Mixer for Band 9 of ALMA," Proc. 18th. ISSTT, 39-43 (2007).

[2] Mena, F. P., Kooi, J. W., Baryshev, A. M., Lodewijk, C. F. J., Hesper, R., Gerlofsma, G., Klapwijk, T. M. and Wild, W., "A Sideband-separating Heterodyne Receiver for the 600-720 GHz Band", IEEE Trans. Microwave Theory Techn., accepted for publication

[3] Tucker, J. R., Feldman, M. J., "Quantum detection at millimeter wavelength” Rev. Mod. Phys. V.57. №4. 10551113 (1985).

[4] Malo, I., Gallego, J. D., Diez, C., López-Fernández, I. and Briso, C., "Improved Multi-octave 3 dB IF Hybrid for Radio Astronomy Cryogenic Receivers", Proc. 20th. ISSTT, 300-306 (2009).

[5] Wild, W. and Payne, J., "Specifications for the ALMA Front End Assembly", (2000) http://www.cv.nrao.edu/ awootten/mmaimcal/asac/ALMA_rx_specs_V10.pdf

[6] Kerr, A. R., Pan, S.K. and Effland, J. E., "Sideband Calibration of Millimeter-Wave Receivers", ALMA memo 357 (2001) http://www.alma.nrao.edu/memos/html-memos/alma357/memo357.pdf 